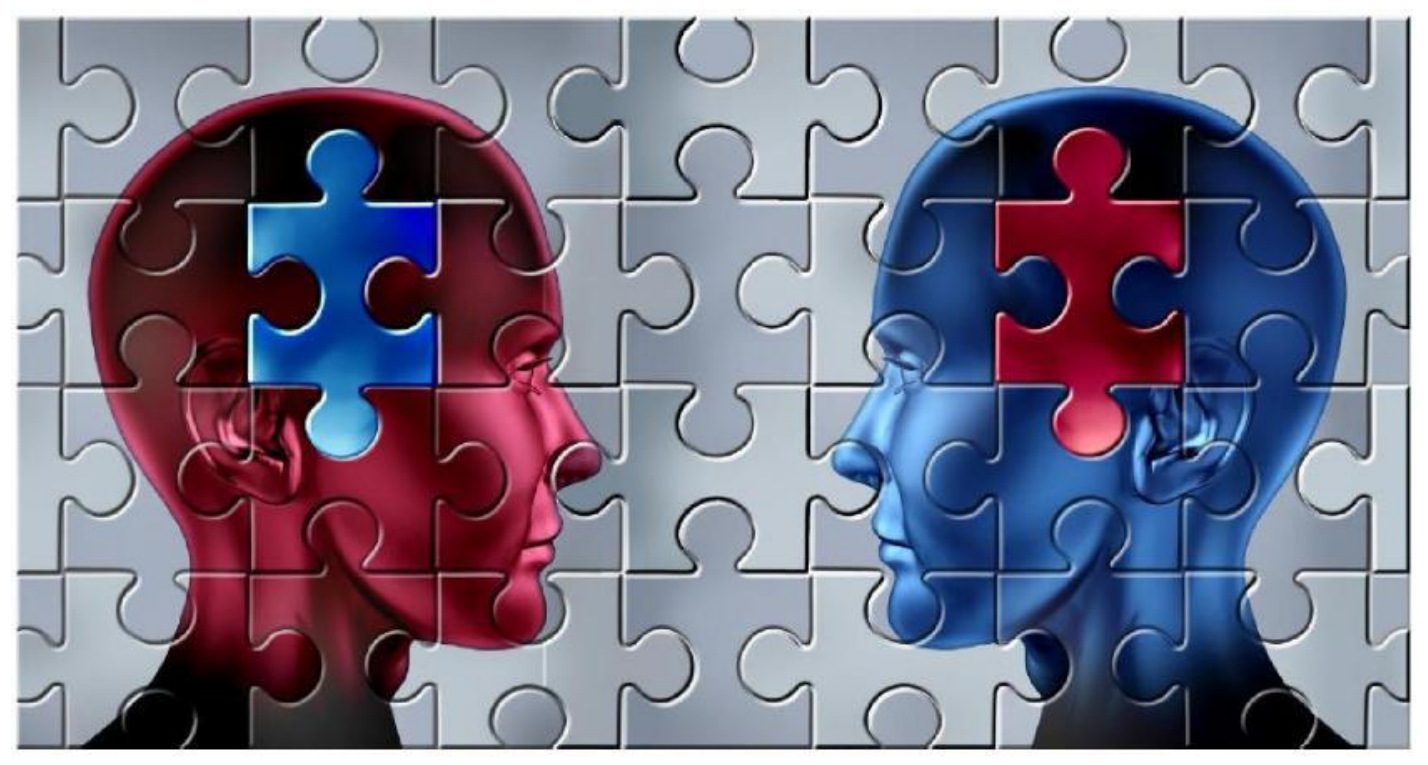

Exchanges: the Warwick Research Journal

\author{
Volume 1, Issue 2, April 2014 \\ http:/ / exchanges.warwick.ac.uk
}

\title{
Creative Research Methods - A Reflective Online Discussion
}

Ruth Leary, Chris Bilton, Hannah Grainger Clemson, Nike Jung, Robert O'Toole, Steve Ranford (University of Warwick) 


\title{
Creative Research Methods -A Reflective Online Discussion
}

Ruth Leary, Chris Bilton, Hannah Grainger Clemson, Nike Jung, Robert O'Toole, Steve Ranford (University of Warwick)

\begin{abstract}
In November 2013 the Institute of Advanced Studies (University of Warwick) hosted a meeting of interdisciplinary colleagues interested in Creative Research Methods. The aspirations were to kick-start the debate at Warwick and create a platform from which researchers can develop projects that embrace new forms of intellectual enquiry and knowledge production. Following the meeting, several of the attendees agreed to develop some of the discussion points and briefly responded to a number of questions in an online document over a period of a few weeks. This paper is the result of that real space and online collaboration.
\end{abstract}

\section{Reflective Discussion}

In November 2013 the Institute of Advanced Studies (University of Warwick) hosted a meeting of interdisciplinary colleagues who had responded to an open invitation from Ruth Leary (Centre for Cultural Policy Studies) to explore their interest in Creative Research Methods. The aspirations were to kick-start the debate at Warwick and create a platform from which researchers can develop projects that embrace new forms of intellectual enquiry and knowledge production. Ruth is currently running an IATL Fellowship initiative, The Mediasmith Project, which is an investigation of transmedia documentary as a research method, and was particularly keen to hear others' views on the use of digital media and technology as research tools. 


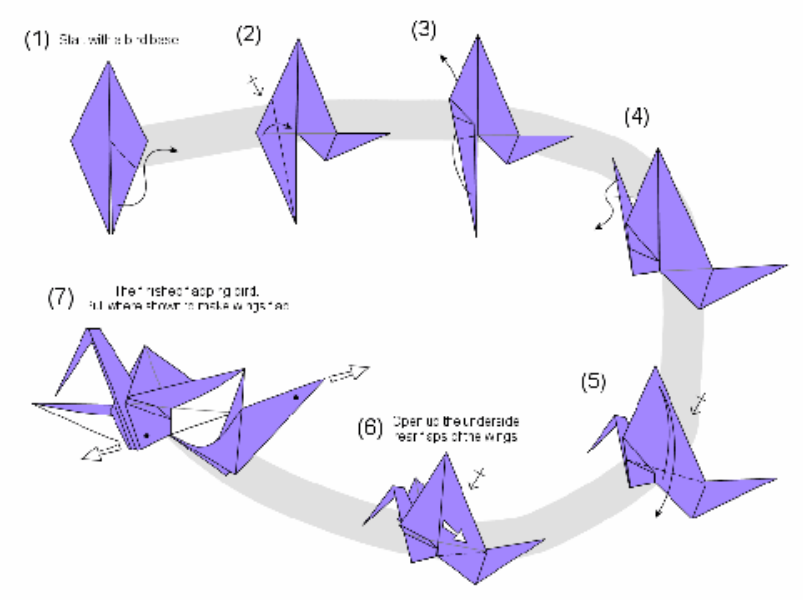

The meeting took the form of small-group brainstorming, feeding back, and then continuing the discussion over some creative activities, including origami. Image: Martin Jackson 2005, http://origami.island-three.net/index.html

Following the meeting, several of the attendees agreed to develop some of the discussion points. In the spirit of experimenting with alternative modes of communication, they briefly responded to a number of questions in an online document over a period of a few weeks, resulting in the following discussion. The content and format has been edited only in a minor way to retain the dialogic style. The group welcomes any comments or feedback from readers - responding either to the questions or the ideas.

Contributors (in alphabetical order): CB - Chris Bilton, Director of the Centre for Cultural Policy Studies ; HGC - Hannah Grainger Clemson, Research Fellow in the Institute of Advanced Study / Centre for Educational Studies; NJ - Nike Jung, PhD student in the Department of Film \& Television; RL - Ruth Leary, Senior Teaching Fellow at the Centre for Cultural Policy Studies ; RO - Robert O’Toole, Senior Academic Technologist \& PhD student, Cultural Policy Studies/Centre for Education Studies/IT Services; SR - Steve Ranford, Senior Academic Technologist for the Faculty of Arts.

\section{When we say 'creative' research methods, do we mean 'arts-based'?}

HGC: I don't think this is exclusively arts-based, as creative to me means trying new forms and approaches to solve problems. I think that when working visually and kinaesthetically one wants to find research methods that suit and embrace that. 
NJ: For me, creative means forgetting the rules - for a moment — to experiment and focus more on the process and the attempt, rather than the result, and this flow-state can obviously also be achieved without any art: it's more related to freedom from confinement but also from immediate criticism.

CB: Creativity is an overused term of course, but in this context I think we're mainly using this as shorthand for anything outside the normal frameworks of academic research and writing. Going a bit further, creativity theory stresses bisociation-combining different frames of reference or thinking styles in unexpected but valuable ways, so I think working across disciplinary boundaries comes into play as well.

HGC: Isn't this quite dangerous, if we have not properly mastered the tools of that other discipline? Perhaps by crossing said boundaries, we are also crossing out of the realm of academia as being specialist knowledge and skills.

$\mathrm{CB}$ : Boundaries are essential to any creative process. 'Thinking outside the box' is not a helpful term here, and expertise within a domain is still important. I'm talking more about combining ways of seeing and thinking, rather than transdisciplinarity. Bisociation could happen by combining different paradigms within an academic discipline, not just by importing some artistic methods from outside.

RL: I worry about not having properly mastered the tools of 'that other discipline'. If we're talking about a transdisciplinary approach, that's when we should be inviting practitioners to work with us to develop approaches that are both authentic (to the discipline) and rigorous. By creative, I'm also thinking about how we facilitate the expression of other forms of intelligence, beyond the linguistic and logical, that more conventional approaches tend to favour.

\section{What has to change in order to legitimise new forms of enquiry?}

RO: We have very few, if any, spaces that can be 'occupied' by a project over a length of time (that is, for longer than a single session on a single day). Creative projects benefit from having a base that can be filled with inspiring and challenging materials (for example, posted on the walls and annotated with post-it notes, and in which prototypes and finished products may be developed, interacted with and tested-out. Not having such spaces significantly affects 
the shape and depth of projects. For example, when a project is hosted in its own space, a wider range of participants are able to access it and contribute. Therefore, this allows a wider range of experiences to be represented in the collection of inspirations/challenges, and gets more people to interact with prototypes. By restricting participation to time-limited slots, the possibility of 'legitimate peripheral participation', with all of its direct and collateral benefits, becomes much less likely. For practitioners of 'participatory design' and 'design thinking' approaches, project spaces are essential. See for example Brown's 2008 paper on 'Design Thinking' for an account of the IDEO 3 Spaces approach. There are also significant similarities between such project spaces and scientific laboratories. This may help in drawing scientists into creative collaborations, working in spaces that are more familiar to them than the traditional Arts seminar.

HGC: Does this come down to academic snobbery and even naivety? In his book, Practice as research in the arts: Principles, protocols, pedagogies, resistances, Nelson (2013) states:

$$
\begin{aligned}
& \text { Limited attention has been paid to the institutional constraints that in some } \\
& \text { instances have hindered the development of PaR [Practice as Research]. These } \\
& \text { range from strong academic traditions which privilege theory, to divisions } \\
& \text { between theory and practice in the very structures of education (university vs. } \\
& \text { art school/conservatoire), and regulatory frameworks which in some instances } \\
& \text { effectively exclude PaR by inscribing 'the scientific method' into research } \\
& \text { regulations. (2013:5) }
\end{aligned}
$$

RO: Where participatory approaches seek to connect with and transform diverse communities (for example, connecting together the Arts and Sciences), project spaces need to be embedded and open. Bjögvinsson, Ehn, and Hilgren (2012) give a good account of this approach, and how they used a set of interconnected embedded design spaces to link together disparate communities. These approaches are essential when addressing complex, ill-defined, multiperspectival, non-linear problems of the kind described by designers as 'wicked problems' (Buchanan 1992). However! This poses a significant challenge to conventional university infrastructures, which are oriented towards disconnected and almost disembodied teaching events. My view (based on my research) is that universities that are able to change their orientation from the generic lecture theatre to the creative project space have a 
significant advantage. Universities that are locked-into the lecture/seminar/own-study triad (building ever bigger lecture theatres) will struggle to adapt to the requirements of a creativedesignerly economy. Drawing upon disciplines that already work in these ways is an essential strategy in achieving this turnaround (Theatre, Creative Writing, Architecture, etc.). But! Online digital spaces, when designed really, really well (and I'm not convinced we have anything good enough yet) could do a similar job.

HGC: As a journal editor, I am constantly encouraging authors to incorporate a range of ways of expressing and sharing data. Publishing online facilitates this and I think if the outputs shift in form, then the enquiries that lead to them will have the freedom to do so.

RL: I agree there could be a degree of academic snobbery and naivety at play here. There is a danger that the academy sees itself as the place of those who think, which does practitionerswho are equally as reflective and intellectually engaged with their work-a huge injustice. Collaborative working and knowledge sharing practices tend to place less emphasis on the role of the expert, which means a rethink of attitudes and priorities. We are already seeing this reflected in the debate about open publishing.

\section{Does the role of the researcher have to change with a more creative methodology?}

NJ: The role of everyone and everything involved would change - and that is scary, and maybe uncomfortable, because it is something new, where the rules and the correct way of proceeding are not yet fixed. And we are trained to avoid 'failure' at all costs.

CB: I think any 'creative' methodology - like creative practice - will involve a bit more risk, putting one's personality on the line and the risk of appearing ridiculous. The approach may be more participatory too with the researcher as orchestrator / facilitator, rather than sole author - a surrender of authority on all sides. And because of the embarrassment potential, the researcher may need to reassure and prompt, encouraging participants to dive in without too much forethought.

SR: Does a pattern of starting by defining the 'research question' hinder the ability for more explorative and creative research methods? Are there other approaches that could be explored that lead to more creative methodologies? Is there institutional pressure for research to fit into 
a nicely framed research question; is it a deeply ingrained habit, or do we need it for our brains to process and function in the research space?

HGC: So are we talking about barriers at an institutional level or an individual researcher level? In my experience there is a challenge in that the kinds of methods we employ often involve both a more subjective placing of the researcher and a re-framing as they mould evidence for dissemination. An example would be working with a group of participants to explore places or experiences through the taking of photographs or devising a performance piece.

RL: Lack of objectivity is a familiar criticism aimed at practice-led and other 'creative' research methods but subjectivity and 'moulding the evidence' is an inherent risk in all research. We are perhaps more practised at controlling for this within more familiar research frameworks. 'Holding' a space that facilitates both exploration and experimentation but also upholds academic values such as rigour and critical analysis is also a challenge. This inevitably necessitates a de-throning of authority but simultaneously escalates the researcher's responsibility as the agent of a process which is unpredictable, and therefore feels riskier for all concerned.

\section{How does your interest in creative research methods capture the spirit of your own pedagogical approach?}

RL: I've been exploring my interest in this area for some time by facilitating open space learning workshops that draw upon kinaesthetic and experiential learning, forum theatre and improvisation techniques. Storytelling, embodiment, liminality and reflective practice are key words for me.

HGC: Key words that spring to my mind are: collaboration; dialogue; narrative; playful.

CB: Drawing an image of organisation, role-playing a decision process or a negotiation, playing with Lego - these slice through the more predictable responses we might normally have in a classroom discussion. It's also an opportunity for students who may not be so confident / articulate verbally. A mix of methods allows different ideas and people to come to the front. I find there's a bit of awkwardness / suspicion at first but once they get going it can flow. Speed and time limits help!

Exchanges: the Warwick Research Journal, 1(2), April 2014 
HGC: I agree that there is always a degree of suspicion at first but we are in the privileged position of having faith in these approaches. My concerns are for a) those educators who have not had these experiences, and b) students (and therefore future researchers) coming through a primary and secondary education system which is in danger of losing such an ethos altogether.

\section{What do 'play' and 'improvisation' mean to you? CB:}

Starting a sentence before you know how it's going to...

RL: I am reminded of Picasso's saying: 'Every child is an artist. The problem is how to remain an artist once he grows up'. Improvisation and play mean (re)connecting and engaging with your inner child.

HGC: Both have a spirit of freedom and of trial and error, where multiple interpretations are expected and accepted. However, I think both are still governed by rules, agreed upon by the participants (even tacitly) to create purposeful working boundaries. There is also a sense of being the audience for each other, rather than a separation. This makes them quite close, personal, and of-the-moment. Actions can be altered 'next time' but the capacity for profound and lasting experiences is still there. The freedom to 'play' in research is great, but I am not sure 'improvisation' is tolerated. I'd be genuinely surprised and delighted to hear of examples.

NJ: Definitely a more interactive, less determined procedure, which involves both mind and emotions.

HGC: At the beginning we discussed how 'creative' does not necessarily mean 'arts-based', however researchers in the arts are already in tune (!) with a more messy process of discovery. In Research Methods in Theatre and Performance, the editors, Baz Kershaw and Helen Nicholson, state in the introduction that:

Getting lost, meeting obstacles or generating disagreement in the methods and methodologies maze are intrinsic to collaboration, but these moments of confusion, dissent or antagonism can be very research-rich (2010:2) 
CB: Getting lost also requires a level of self-confidence. Or in the case of our students, a trust in the educational process and in us as educators, believing that their confusion is a creative journey and not merely confusing!

\section{Are we using technological tools effectively enough?}

HGC: I think there is scope for more integration.

SR: There is a lot of potential to be explored in how technical tools can be integrated into creative research methods. In relation to both research data sources, that people are using to discover insight, and the ways that data is being manipulated and visualised. I'd argue that the techniques and mind-sets that are needed to effectively exploit this potential require interdisciplinary skillsets, and are in short supply.

RL: I completely agree - integration is key. I share the frustration that I have a good sense of the potential but lack the technical skills to realise it.

HGC: But is it that our generation ('surviving school without Google') do not possess the skills or is it that human superiority and belief in our own bodies and minds as a near-perfect window on the world prevents us ever accepting it—perhaps both causing a vicious circle? Is this where the arts fall down compared to the sciences, who have long-embraced technology?

CB: There is a danger of fetishising technology, especially with the emphasis on 'digital tools' in education and the arts. Old technologies (storytelling, visualisation) can trigger new ideas.

RL: I think we need to be careful here. Artists have often been the first to exploit technology in new and unimagined ways. However, using technology gratuitously has less value than not using it at all; our challenge is to work out what it can add, not necessarily what it can substitute for. Video didn't kill the radio star! 


\section{What do we expect or want from our research audiences? NJ: Ideally, to}

give space and time as if it is the first time. Which goes both ways.

CB: Ideally to be more interested in the subject than in the method we are using to find out about it: open minds and curiosity from the audiences as well as researchers, tolerance of risk and failure, all the things you'd hope to encounter in an audience for experimental creative practice.

HGC: Less snobbery from academics; more listening by the government; increased confidence in the general public.

\section{What place have emotion and lived experiences in research methodology?}

NJ: They are in fact in there all the time, but usually not admitted to because we still work with a body-mind split.

RL: I agree. I find this mind-body split deeply problematic and have been able to draw on my background in movement and dance to some extent, but there is scope for more. Digital storytelling and visual sociology techniques are helping us realise the value of emotion and lived experience and there is an emerging field of research in memory and affect in digital media studies.

HGC: Performance Ethnography (see Denzin 2003 amongst others) interests me because it directly gets to grips with the lived experience and 're-lives' it as a way of exploring and understanding it, but in a more objective fashion. It is an alternative to the singular view problem I described above.

\section{Conventional research can be democratic. Is creativity just less}

'disciplined'? NJ: I don't think these things are mutually exclusive or opposed.

SR: I find I'm the most creative when responding to the challenge of enabling constraints, rather than in a vacuum of rules. Is creativity already the process of adding discipline to imagination? 
CB: I agree that there is potentially a false dichotomy here. We tend to work more creatively within constraints than without them. And of course one could do a lot of the things we've discussed here within the constraints of 'conventional' research.

HGC: If we are being 'creative' then being new is perhaps going to be less structured initially. If we are being 'playful' then rules are more flexible and boundaries are blurred. In the other sense of 'discipline', the more we share of our methodologies, the more of a CRM 'discipline' we can create in academia.

RL: Maybe it's a question of timing and when to apply the rules rather than a question of being less 'disciplined'; purposeful play as opposed to play for play's sake. In my experience much of the value is realised through structured reflection afterwards - the process alone is not enough.

\section{What are the possibilities for creative research methods to create higher charged, political spaces that stimulate debate?}

NJ: At this moment that seems too early to tell.

RL: It seems as if the debate is gathering momentum although until now it has mainly been the preserve of practice-based humanities disciplines. Interest in alternative research methods probably needs to reach some kind of cross-disciplinary tipping point and I think it's likely to be the use of new technological tools that will precipitate this.

\section{Notes}

The 2014 International Federation for Theatre Research World Congress will be hosted by the School of Theatre, Performance and Cultural Policy Studies at the University of Warwick, 28 July-1 Aug, when the 'Performance as Research' Working Group will also be meeting. For more information, visit http://iftr2014warwick.org/

Ruth Leary is leading an IATL funded Fellowship The Mediasmith Project exploring transmedia documentary as a creative research method. For more information, go to http://www.warwick.ac.uk/go/mediasmith 


\section{References}

Brown, T. (2008), 'Design Thinking', Harvard Business Review, June 2008, 84-95. Accessed online at http://www.ideo.com/images/uploads/thoughts/IDEO HBR Design Thinking.pdf

Bjögvinsson, E., P. Ehn, and P-A. Hillgren (2012), 'Design Things and Design Thinking: Contemporary Participatory Design Challenges', Design Issues, 28 (3), 101116. Accessed online at http://www.mitpressjournals.org/doi/abs/10.1162/DESI a $\underline{00165}$

Buchanan, R. (1992), 'Wicked Problems in Design Thinking', Design Issues, 8 (2), 5-21. Accessed online at http://www.jstor.org/stable/1511637

Denzin, N.K. (2003), Performance Ethnography: Critical Pedagogy and the Politics of Culture, London: SAGE

Kershaw, B. and Nicholson, H (2010), Research Methods in Theatre and Performance, Edinburgh: Edinburgh University Press

Nelson, R (ed) (2013), Practice as research in the arts: Principles, protocols, pedagogies, resistances, Basingstoke: Palgrave Macmillan 